\title{
Chapter 36 \\ Biochemical Evidence of Convergent \\ Evolution of Aposematic Coloration \\ in Thorny, Spiny and Prickly Plants
}

A very strong, indirect evidence for the operation of aposematic coloration in thorny, spiny and prickly plants and its convergent evolution is the fact that conspicuous thorn, spine and prickle coloration is found in Angiosperm taxa that have mutually exclusive biochemical pathways of coloration. For instance, taxa belonging to the Caryophyllales (e.g., Cactaceae, Caryophyllaceae, Amaranthaceae) produce yellow, orange, red, purple and black pigments via the betalain pathway (Stafford 1994). Most other Angiosperm families use anthocyanins or carotenoids for similar patterns of coloration. The fact that spines of cacti are usually conspicuous because of their coloration (Lev-Yadun 2001), commonly including yellow, orange, red and black coloration resulting from betalain derivatives, indicates that this group of pigments is involved, among their various important physiological functions (e.g., Jain and Gould 2015), in aposematic coloration. By contrast, in Rosaceae, Asteraceae and Fabaceae as well as in many other Angiosperm families that use anthocyanins for orange, pink, red, blue, purple and black coloration of thorns, spines and prickles, the chemical origin of the aposematic coloration (LevYadun 2001, 2006a; Lev-Yadun and Gould 2009) is different. It seems therefore that the aposematic coloration of thorny, spiny and prickly plants is a good case of convergent evolution. 